\title{
Effect of Cold Spraying Parameters and Their Interaction an Hydroxyapatite Deposition
}

\author{
R. P. Singh ${ }^{1 \dagger}$, U. Batra ${ }^{2}$ \\ ${ }^{1}$ Department of Mechanical Engineering, Chitkara University, Rajpura, Punjab, India-140401 \\ ${ }^{2}$ Department of Materials and Metallurgy, PEC University of Technology, Chandigarh, India-160012 \\ †Corresponding Author Email: er.ravinderpalsingh@gmail.com
}

(Received April 24, 2012; accepted September 20, 2012)

\begin{abstract}
Cold spraying has been effectively investigated for spraying of various metals, alloys and composites. Coating of bioceramics like Hydroxyapatite (HAP) using thermal spraying has been investigated extensively, but there is a dearth of research related with HAP deposition using cold spraying. The relative percentage contribution of five important cold spraying parameters, their optimum combination and expected HAP particle velocity at optimum combination have been extensively discussed by the author, but no researcher reported the interactional effect of cold spraying parameters on HAP particle velocity. This paper examined the severity index of interactional effect of five important factors of cold spraying viz. gas type, gas inlet pressure, gas inlet temperature, particle size and particle temperature on HAP particle velocity. Most influential factor pairs and their influence on HAP particle velocity have been discussed with respect to various cold spraying gases viz. air, hydrogen, nitrogen and helium. In addition, CFD gas pressure and temperature distributions along the nozzle length and their mathematical validation have also been undertaken in the present paper.
\end{abstract}

Keywords: CFD, Cold spraying, Taguchi, Particle velocity.

\section{NOMENCLATURE}

$$
\begin{array}{ll}
\mathrm{T}_{\mathrm{o}} & \text { inlet temperature of gas } \\
\mathrm{T} & \text { exit gas temperature } \\
\gamma & \text { adiabatic index }
\end{array}
$$

$\begin{array}{ll}\mathrm{M} & \text { gas Mach number } \\ \mathrm{P} & \text { exit gas pressure } \\ \mathrm{P}^{*} & \text { throat gas pressure }\end{array}$

\section{INTRODUCTION}

Cold spraying is a new emerging spray technology (Singh, 2011; and Li, 2006) in which coating is formed through the plastic deformation of spray particles that are in a completely solid state during impact (Kosarev, 2002; and Dykhuizen, 1998). Most metals and their alloys can be deposited by cold spraying ( $\mathrm{Li}, 2006$; and Alkimov, 1990). Even cermets (Lima, 2002) can be deposited and ceramic particles $(\mathrm{Li}, 2004)$ can be embedded into the metal substrate to form a thin layer coating ( $\mathrm{Li}, 2004)$.

It has been widely accepted that particle velocity prior to impact is one of the most important parameters in cold spraying (Li, 2006; Lupoi, 2010; Katanoda, 2008; Raletz, 2006; Tabbara, 2010; and Li, 2004) and determines whether deposition of a particle or erosion of substrate occurs on the impact of a spray particle. Generally for a given material, there exists a critical velocity (Katanoda, 2008; Li, 2004; and Papyrin, 2001) for its successful deposition on the substrate. Only those particles achieving the particle velocity higher than the critical one can be deposited to produce a coating ( $\mathrm{Li}$, 2004, 2006; and Mulligan, 2009). The critical velocity is associated with properties of the spray material (Mulligan, 2009; Steenkiste, 1999; and Staltenhoff , 2002) and physical properties of the driving gas, its operating pressure and temperature, as well as the nozzle design of the spray gun (Dykhuizen, 1998; Li, 2004; Gilmore, 1999; Steenkiste, 2002; and Champagne, 2010). The characteristics of powder such as density (Raletz, 2006; and Tabbara, 2010) particle size (Dykhuizen, 1998; Tabbara, 2010; Li, 2004; and Steenkiste, 2002), morphology (Li, 2004, 2003) and temperature (Dykhuizen, 1998) also influence the particle acceleration and subsequent deposition. Accurate knowledge of particle velocity is thus critical to the prediction of the quality of the anticipated cold spray applications.

The progress in the computational fluid dynamics (CFD) has made it possible to simulate gas-solid two phase flow precisely (Li 2004, 2006). Several reports 
have shown the feasibility to use CFD approach to obtain reasonable results, Gilmore (1999), Jodoin (2006) as cited in Li (2006). FLUENT software package has been proven to be reliable for modeling the gas flow in a Laval nozzle (Jodoin, 2002) and reported practically validated results (Tabbara, 2010; Li, 2004, 2006; and Sanjay, 2010).

Hydroxyapatite (HAP) has been an extensively accepted bio-ceramic used for implant applications in orthopedics, maxillofacial surgery and dental implants (Thamaraiseli, 2004). Plasma and High Velocity Oxy Fuel (HVOF) spraying techniques have been extensively used for HAP deposition despite many limitations due to the involvement of high temperature. On the other hand, cold spraying uses high velocity rather than high temperature to produce coatings and thereby avoid/minimize main deleterious high temperature reactions that occur in Plasma and HVOF (Karthikeyan). In addition, to the best of our knowledge, report of successful fabrication of HAP coating by cold spraying technology is not available in open literature; hence needs attention for research. Author has evaluated the individual effects of varied cold spraying parameters on HAP particle velocity viz. types of gas, gas pressure, gas temperature, particle size and particle temperature with an aid of CFD, mathematical and Taguchi technique (Singh, 2011), but the detailed analysis of interaction between the cold spraying parameters is yet to be ascertained which will in turn be used to evaluate the combined effects of cold spraying parameters and their relation with HAP particle velocity.

Taguchi technique is an experimental design optimization technique (Tsa, 2008; Roy, 2001; and Senthilkumar, 2010) which use standard 'Orthogonal Arrays' for forming a matrix of experiments in such a way to extract the maximum important information with minimum number of experiments. Using Taguchi techniques, the number of parameters can be tested at a time with probably least number of experiments as compared to any of the other experimental optimization techniques. Moreover, the technique provides all the necessary information required for optimizing the problem. The main advantage of Taguchi Techniques is not only the smallest number of experiments required but also the best level of each parameter can be found and each parameter can be shared towards the problem separately. The main steps of Taguchi method are determining the quality characteristics and design parameters necessary for the product/process, designing and conducting the experiments, analyzing the results to determine the optimum conditions and carrying out a confirmatory test using the optimum conditions (Senthilkumar, 2010).

The present paper reports the most influential interacted cold spraying parameter combinations, their relative percentage contributions, and their combined effect on simulated HAP particle velocity. In addition, numerically simulated gas pressure and temperature distribution along the nozzle length have been validated mathematically.

\section{Methodology}

This study has been conducted in following phases (Singh, 2011):

1) Selection of Factors, Levels and orthogonal Array

2) FLUENT Modeling and Numerical Simulations

3) Taguchi Methodology

4) Mathematical Validation

\subsection{Selection of Factors, Levels and Orthogonal Array}

Three factors related with gas properties viz. gas type, gas inlet pressure (MPa), gas inlet temperature $(\mathrm{K})$ and two factors related with spraying particles viz. particle diameter $(\mu \mathrm{m})$ and particle temperature $(\mathrm{K})$ were selected for the study. Further four levels were chosen for each factor and have been reported elsewhere (Singh, 2011). L-16 orthogonal array was formulated using Taguchi approach.

\subsection{FLUENT Modeling and Numerical Simulations}

Gambit 2.3.16 package was used to model the 2D axis symmetric convergent-divergent (CD) nozzle. Modeling, mesh generation, mesh quality, boundary conditions and allocation of fluid were done in Gambit environment. Numerical simulation was carried out by using the commercial software FLUENT to determine the flow field of the driving gas inside and outside the nozzle and subsequently heating and accelerating of particles in the cold spray. Schematic diagram, meshed model of axis-symmetric convergent-divergent nozzle, properties of HAP powder, boundary conditions and governing equations have been discussed in details elsewhere (Singh, 2011).

\subsubsection{Governing Equations}

The equations governing the steady-state gas flow are the mass, momentum and energy conservation equations. To account for turbulence in the flow, a k- $\varepsilon$ turbulence model was used. The flow being compressible, the gas mass density was not constant, and one more equation was used to close the system of equation: the perfect gas law.

\subsection{Taguchi Methodology}

Qualitek-4 software was used for complete analysis of nozzle exit particle velocity. Average, standard deviation, main average effects, analysis of variation (ANOVA), optimum conditions and expected optimum particle velocity was calculated and plotted. Confirmation test at optimum conditions was also conducted and have been reported elsewhere (Singh, 2011). Interaction among factors was not considered in study (Singh, 2011) which becomes the objective of the present paper. Effect of most influential factor pairs on HAP particle velocity from nozzle exit was evaluated and plotted with respect to various gases i.e. air, nitrogen, helium and hydrogen. Interaction severity index of interacted factors was also evaluated. 


\subsection{Mathematical Validation}

Numerically simulated optimal particle velocity behavior was validated with one dimensional isentropic (adiabatic and frictionless) mathematical equation (Singh, 2011). Assumptions, gas and particle velocity equations have been discussed in details (Singh, 2011). In the present study, CFD evaluated gas pressure and temperature distribution along the nozzle length was plotted and mathematically validated for generous understanding of probable gas flow behavior in nozzle. With the Mach number known along the nozzle length, gas pressure and temperature distribution along the nozzle length was calculated by using following Eqs. (1) and (2):

$$
\frac{T_{0}}{T}=1+\left(\frac{\gamma-1}{2}\right) M^{2}
$$

where $T_{o}$ is inlet temperature of gas, $T$ is exit gas temperature, $\gamma$ is adiabatic index and $\mathrm{M}$ is gas Mach number inside nozzle. From Eq. (1), exit temperature (T) can be calculated with the Mach number known (M)

$$
\frac{P}{P^{*}}=\left[\frac{(\gamma+1)}{2+(\gamma-1) M^{2}}\right]^{\frac{\gamma}{\gamma-1}}
$$

where $\mathrm{P}$ is exit gas pressure, $\mathrm{P} *$ is throat gas pressure, $\gamma$ is gas adiabatic index and $\mathrm{M}$ is gas Mach number. From Eq. (2), exit gas pressure was calculated along the cross-sections of nozzle length and plotted, after calculating the gas pressure at nozzle throat.

\section{RESULTS AND DISCUSSION}

\subsection{Interaction Severity Index}

After calculating the average effect of each individual factor on HAP particle velocity, optimum conditions and expected particle velocity at optimum conditions (Singh, 2011), interaction among factors and their respective severity index was also calculated using Taguchi approach. Presence of interaction and their severity index are enumerated in Table 1.

Table 1 Interacted Factor Pairs and Corresponding Severity Index

\begin{tabular}{|c|c|c|}
\hline $\begin{array}{c}\text { S. } \\
\text { No. }\end{array}$ & Interacted Factor Pair & $\begin{array}{c}\text { Severity } \\
\text { Index }\end{array}$ \\
\hline 1 & $\begin{array}{c}\text { Gas Pressure X Particle } \\
\text { Diameter }\end{array}$ & 65.48 \\
\hline 2 & $\begin{array}{c}\text { Gas Temperature X Particle } \\
\text { Diameter }\end{array}$ & 56.05 \\
\hline 3 & $\begin{array}{c}\text { Gas Temperature X Particle } \\
\text { Temperature }\end{array}$ & 35.19 \\
\hline 4 & $\begin{array}{c}\text { Particle Diameter X Particle } \\
\text { Temperature }\end{array}$ & 18.71 \\
\hline 5 & $\begin{array}{c}\text { Gas Pressure X Particle } \\
\text { Temperature }\end{array}$ & 18.34 \\
\hline
\end{tabular}

To understand comprehensively the effect of cold spraying factors on HAP particle velocity, it is mandatory to recognize the interaction among the spraying factors, if any. Five interacting pairs were found to considerably influence HAP particle velocity in cold spraying. Gas inlet pressure combined with particle diameter was found to maximally affect the particle velocity followed by other pairs as shown in Table 1. Interaction severity index of gas inlet pressure combined with particle diameter and gas inlet temperature combined with particle diameter affect the particle velocity more than $50 \%$. Gas pressure, gas temperature and particle diameter have been reported by many researchers (Champagne, 2010; Li, 2004; and Jodoin, 2006, 2002) to be the main influential factors affecting the particle velocity.

\subsection{Effect of Spraying Factors on HAP Particle Velocity}

Effect of most influential interacted factor pairs, Table 1, on HAP particle velocity has been shown in Fig. 1-5. Behavior of various cold spraying gases i.e. air, nitrogen, helium and hydrogen were analyzed. Influence of gas pressure combined with particle diameter on HAP particle velocity is shown in Fig.1.

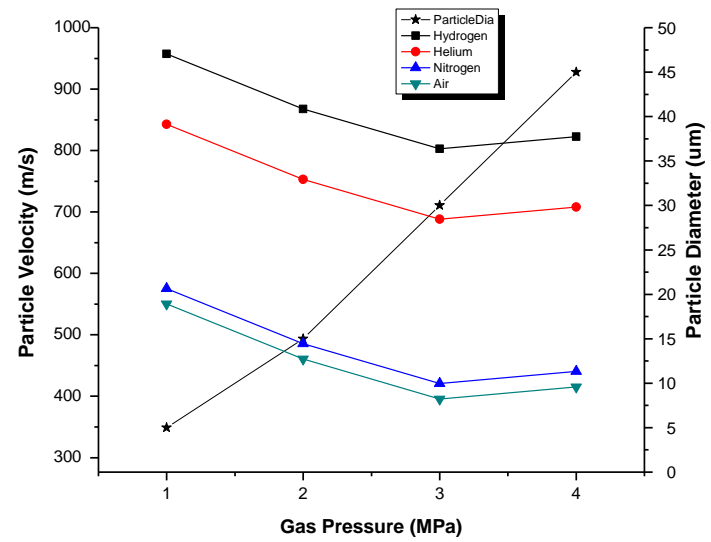

Fig. 1. Influence of Gas Pressure and Particle Diameter on HAP Particle Velocity

Particle velocity was found to decrease with the increase of gas pressure and particle size. At a gas pressure of $1 \mathrm{MPa}$ and particle size of $5 \mu \mathrm{m}$, the particle velocity was found to be maximum and it decreased linearly with the increase of pressure up to $3 \mathrm{MPa}$ and particle diameter of $30 \mu \mathrm{m}$. Particle velocity was observed to be maximum with the usage of hydrogen gas followed by helium, nitrogen and air respectively. Many investigations have revealed to use the small particle size of spraying powder to achieve the highest particle velocity. Also, hydrogen and helium have been reported to produce the maximum particle velocity due to their low atomic weight as compared to nitrogen and air (Jodoin, 2002; and Katanoda, 2008).

Influence of gas temperature combined with particle diameter on HAP particle velocity is shown in Fig. 2. As the gas temperature and particle diameter increased, particle velocity was observed to decrease. Between gas temperature of $373-573 \mathrm{~K}$ along with particle diameter ranges from $15-30 \mu \mathrm{m}$, particle velocity was observed to almost remain same. Highest particle velocity was examined at the smallest particle diameter of $5 \mu \mathrm{m}$ with a gas temperature of $303 \mathrm{~K}$ with various gases. Highest particle velocity was observed with the usage of hydrogen gas followed by helium, nitrogen and air. Many investigations have revealed to use the small 
particle size of spraying powder to achieve the highest particle velocity. Also, hydrogen and helium has been reported to produce the maximum particle velocity.

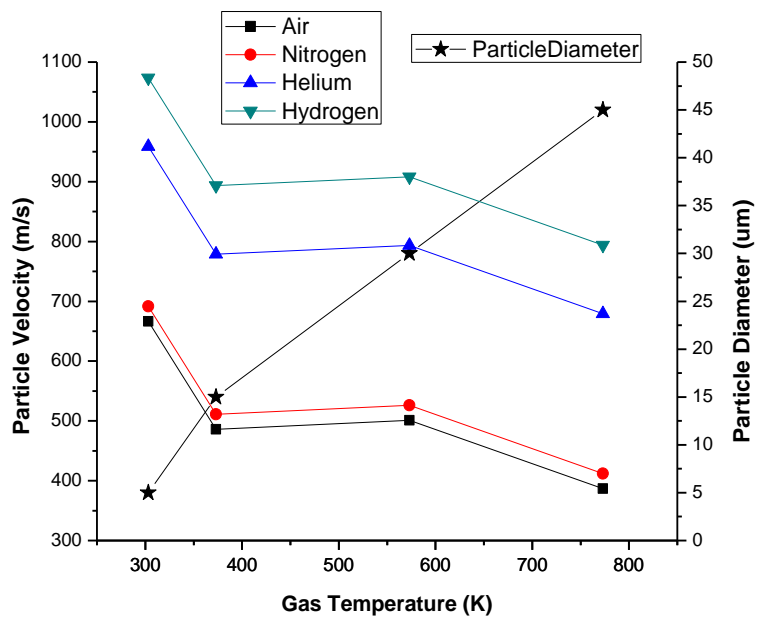

Fig. 2. Influence of Gas Temperature and Particle Diameter on HAP Particle Velocity

Comparison of Fig. 1 and Fig. 2 concludes that the HAP particle velocity is inversely proportional to particle size, despite the increase of gas pressure and gas temperature.

Influence of gas temperature and particle temperature on HAP particle velocity is shown in Fig. 3. It shows that with the increase of gas and particle temperature, particle velocity increased. Hence comparison of Fig. 2 and Fig. 3 establishes that to increase the HAP particle velocity, along with the increase of gas temperature, increase of particle temperature is also mandatory at constant particle size. Similar directly proportional effect of gas and particle temperature at constant particle size on particle velocity has already been experimentally supported by many investigators (Champagne, 2010; and Li, 2004). Maximum particle velocity can be achieved at a maximum particle temperature with the usage of hydrogen followed by helium gas.

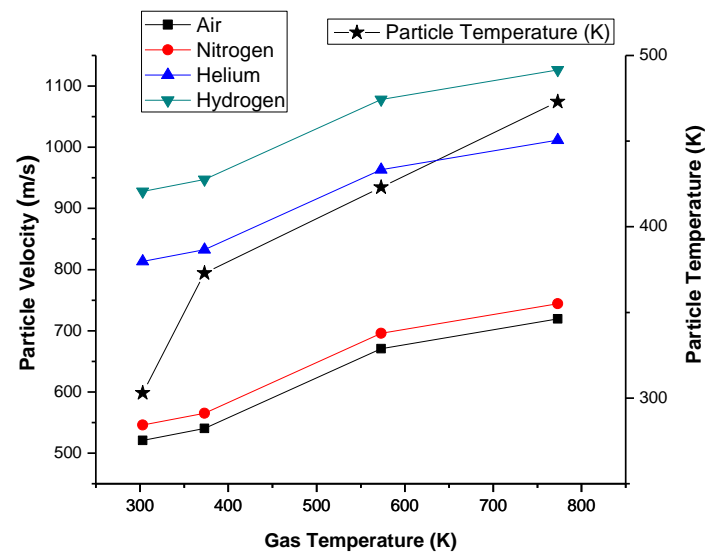

Fig. 3. Influence of Gas Temperature and Particle Temperature on HAP Particle Velocity

Now, it has been well established from Fig. 2 and Fig. 3 that HAP particle velocity increases only, if along with the increase of gas temperature; particle temperature is also increased. This behavior is only valid for particles having uniform/constant size. This latter behavior is further confirmed by Fig. 4 which suggests that even on the increase of particle temperature, particle velocity will not increase and rather it will decrease, if particle size is increased. This analysis further firms the previous conclusion of decrease of particle velocity with the increase in particle size. It is now crystal clear that particle size produces the dominating inversely proportional effect on HAP particle velocity.

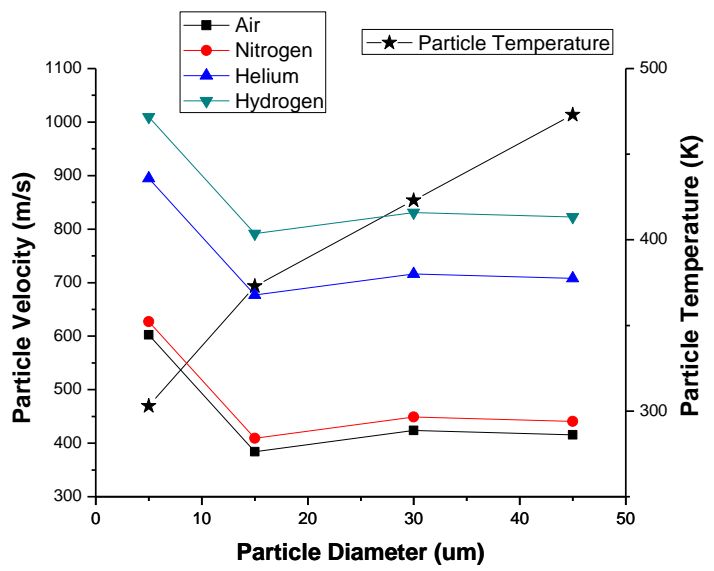

Fig. 4. Influence of Particle Diameter and Particle Temperature on HAP Particle Velocity

Increase of gas pressure and particle temperature consequently increased the particle velocity as shown in Fig. 5 and has been reported by many investigations (Champagne, 2010; and Li, 2004). Hydrogen followed by helium gas furnished the maximum particle velocity at highest gas pressure and particle temperature.

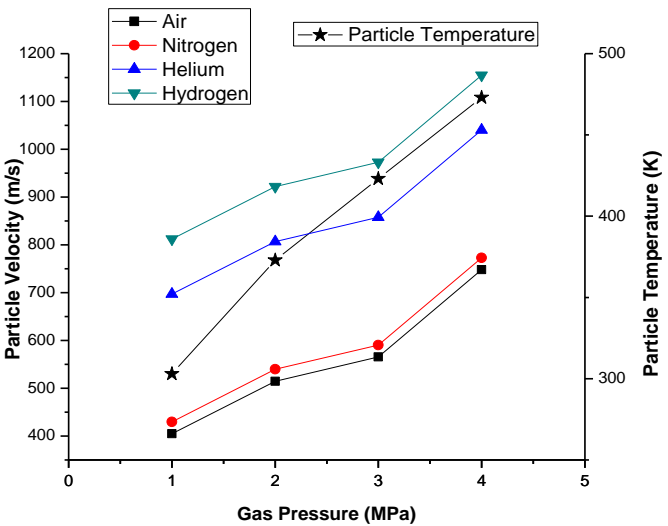

Fig. 5. Influence of Gas Pressure and Particle Temperature on HAP Particle Velocity

\subsection{CFD Gas Pressure and Temperature Distribution and Mathematical Validation}

Figure 6 shows the comparison of Computational Fluid Dynamics (CFD) and mathematically (1-D) evaluated gas pressure distribution of optimized gas i.e Hydrogen along the nozzle length. CFD gas pressure distribution confirmed to be less than the mathematically calculated gas distribution range as mathematical formula doesn't take into consideration the effect of friction between nozzle wall and gas layer. From inlet to exit, gas pressure was reduced due to its expansion in convergent 
divergent nozzle. Near the nozzle exit, fluctuation in pressure was also observed.

Figure 7 shows the comparison of Computational Fluid Dynamics (CFD) and mathematically (1-D) evaluated

gas temperature distribution of optimized gas i.e Hydrogen along the nozzle length. CFD evaluated temperature was observed to be less than the mathematically calculated gas temperature, but pattern of distribution observed to be same along the nozzle length. From inlet to exit, gas temperature was reduced due to its expansion in convergent divergent nozzle.

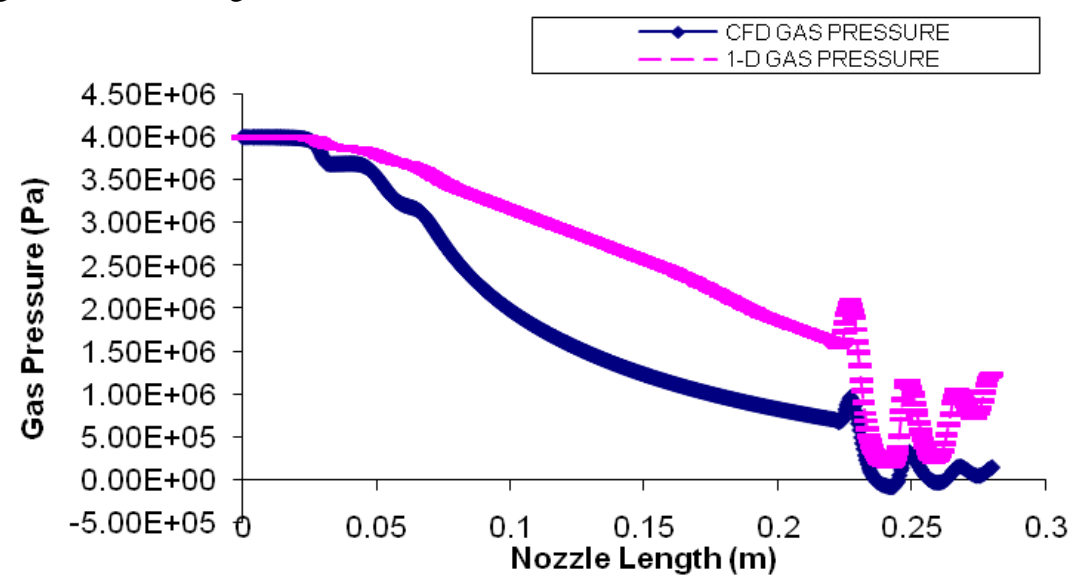

Fig. 6. Comparison of CFD and Mathematical Gas Pressure Distribution in Optimized Nozzle

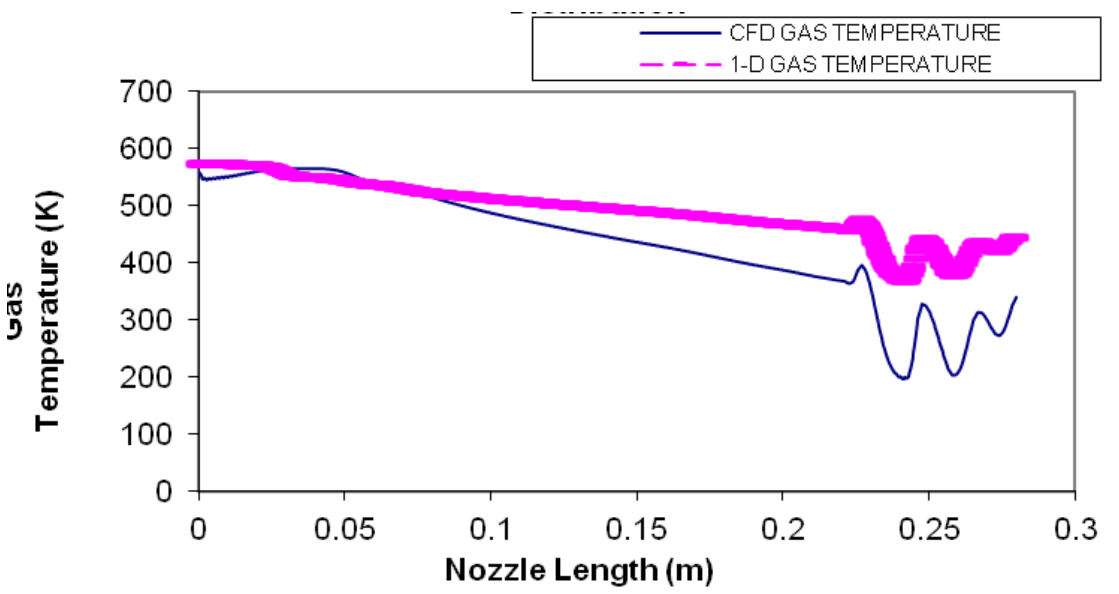

Fig. 7. Comparison of CFD and Mathematical Gas Temperature Distribution in Optimized Nozzle

\section{Conclusions}

1. Many investigations only enumerated the influential factors and corresponding levels, but none reported their interactional effect on particle velocity. In the present study, interactions between various cold spraying parameters were studied using Taguchi approach. In addition, severity index of interacting factors were also presented. Gas pressure combined with particle diameter factors were observed to affect the particle velocity maximally followed by other combinations.

2. Increase of HAP particle diameter was found to decrease the particle velocity and its influence was found to be more than the respective influences of gas pressure, gas temperature and particle temperature.

3 . Increase of gas pressure and particle temperature was found to increase the particle velocity.
4. CFD and mathematically calculated Hydrogen gas pressure and temperature distribution patterns was found to be similar and decreased from inlet to exit along the nozzle length. The main reason can be an expansion of hydrogen gas within the nozzle. Similar distribution patterns also confirmed the correctness of simulation procedure and applied boundary conditions in FLUENT software.

\section{REFERENCES}

Alkimov, A.P., V.F. Kosarev and A.N. Papyrin (1990). A Method of Cold Gas Dynamic Deposition, Dokl. Akad. Navk SSSR, 318(5), 1062-1065.

Champagne, V.K., D.J. Helfritch, S.P.G. Dinavahi, and P.F. Leyman (2010). Theoretical and Experimental Particle Velocity in Cold Spray, Journal of Thermal Spray Technology, Published online. 
Dykhuizen, R.C. and M.F. Smith (1998). Gas Dynamic Principles of Cold Spray, Journal of Thermal Spray Technology, 7(2), 205-212.

Fluent 2000a. Gambit 2.3.16. Lebanon, NH: FLUENT, Inc.

Gilmore, D.L., R.C. Dykhuizen, R.A. Neiser, T.J. Roemer, and M.F. Smith (1999). Particle Velocity and Deposition Efficiency in the Cold Spray Process, Journal of Thermal Spray Technology, 8(4), 576-582.

Jodoin, B., F. Raletz, and M. Vardelle (2006). Cold Spray Modeling and Validation using an Optical Diagnostic Method, Journal of Surface and Coating Technology, 200, 4424-4432.

Jodoin, B. (2002). Cold Spray Nozzle Mach Number Limitation, Journal of Thermal Spray Technology, 11, 496-507.

Karthikeyan, Journal of Cold Spray Technology, International and USA efforts, Available at www.google.co.in.

Katanoda, H. (2008). Numerical Simulation of Temperature Uniformity within Solid Particles in cold Spray, Journal of Solid Mechanics and Materials Engineering, 2(1), 58-69.

Kosarev, V.F., S.V. Klinkov, A.P. Alkhimov, and A.N. Papyrin (2002). On Some Aspects of Gas Dynamic of the Cold Spray Process, Journal of Thermal Spray Technology, 12(2), 265-281.

Li, W.Y., H. Liao, G. Douchy, and C. Coddet (2006). Optimal Design of a Cold Spray Nozzle by Numerical Analysis of Particle Velocity and Experimental Validation with 316L Stainless Steel Powder, Journal of Materials and Design, 28, 2129-2137.

Li, C. J., W.Y. Li, and H. Liao (2006). Examination of the Critical Velocity for Deposition of Particle in Cold Spraying, Journal of Thermal Spray Technology, 15(2), 212-222.

Li, C.J., G.J. Yang, X.C. Huang, W.Y. Li, and A. Ohmori, (2004). Formation of $\mathrm{TiO}_{2}$ Photocatalyst through Cold Spraying, Journal of Thermal Spray, Advances in Technology and Application, ASM International, 345-349.

Li, W.Y. and C.J. Li (2004). Optimal Design of a Novel Cold Spray Gun Nozzle at a Limited Space, Journal of Thermal Spray Technology, 14(3), 391-396.
Li., C.J., and W.Y. Li (2004). Optimization of Spray Conditions in Cold Spraying based on the numerical analysis of particle velocity, Journal of Transactions of Non- Ferrous Materials Society of China, 14(2), 43-48.

Li, C.J. and W.Y. Li (2003). Deposition Characteristics of Titanium coating in cold Spraying, Journal of Surface and Coating Technology, 167(2-3), 278283.

Lima, R.S., J. Karthikeyan, C.M. Kay, J. Lindamann, and C.C. Berndt (2002). Microstructural Characteristics of Cold-Sprayed Nanostructured WC-CO coatings, Journal of Thin Solid Films, 416(1-2), 129-135.

Lupoi, R. and W. O'Neill (2010). An investigation of Powder Stream in Cold Gas Spray (CGS) Nozzles. Proceedings of $5^{\text {th }}$ European Conference on Computational Fluid Dynamics (ECCOMAS CFD 2010), Lisbon, Portugal.

Mulligan, P., J. Smith, and J. Stokes (2009). Development of a Cold Flow deposition Process for the Application of Coatings using the HVOF Spray Process, The Arabian Journal for Science and Engineering, 34(1C), 89-99.

Papyrin, A. (2001). Cold Spray Technology, Journal of Advanced Material Process, 159, 49-51.

Raletz, F., M. Vardelle, and G. Ezo'O (2006). Critical Particle Velocity under Cold Spray Condition, Journal of Surface and Coating Technology, 201, 1942-1947.

Roy, R.K. (2001). Design of Experiments Using the Taguchi Approach, New York, USA, John Wiley \& Sons.

Sanjay, P., N.S. Mahesh, and S.K. Kumar (2010). Computational and Heat Transfer Analysis of convergent Nozzle used for Gas Atomization of Liquid Metals, International Journal of Dynamics of Fluids, 6(2), 161-179.

Senthilkumar, R, S. Vaidyanathan, B. Sivaraman (2010). Thermal analysis of heat pipe using Taguchi method, International Journal of Engineering Science and Technology, 2(4), 564-569.

Singh, R.P. (2011). Numerical Evaluation, optimization and Mathematical Validation of Cold Spraying of Hydroxyapatite using Taguchi Approach, International Journal of Engineering Science and Technology, 3(9), 7006-7015. 
R. P. Singh and U. Batra / JAFM, Vol. 6, No. 4, pp.555 -561, 2013.

Staltenhoff, T., J. Voyer, and H. Kreye (2002). Cold Spraying: State of the Art and Applicability, International Thermal Spray Conference, 2002, 366-374.

Steenkiste, T.H., J.R. Smith, and R.E. Teets (2002). Aluminum Coatings via Kinetic Spray with relatively large powder particles, Journal of Surface Coating Technology, 154(2-3), 237-252.

Steenkiste, T.H.V., J.R. Smith, R.E. Teets, J.J. Moleski, D.W. Goskiewicz, R.P. Tison, D.R. Marantz, K.A. Kowalsky, W.L. Riggs, P.H. Zajchowski, B. Pilsner, R.C. McCune, and K.J. Barnett (1999). Kinetic Spray Coatings, Journal of Surface Coating Technology, 111(1), 62-71.
Tabbara, H., S. Gu, D.G. McCartney, T.S. Price, and P.H. Shipway (2010). Study on Process Optimization of Cold Gas Spraying, Journal of Thermal Spray Technology, Published online.

Thamaraiseli, T.V. and S. Rajeswari, (2004). Biological Evaluation of Bioceramic Materials-A Review, Trends in Biomaterials and Artificial Organs, 18(1), 9-17.

Tsa, F.C., B.H. Yan, C.Y. Kuan, and F.Y. Huang (2008). Taguchi and experimental investigation into the optimal processing conditions for the abrasive jet polishing of SKD61 mold steel, International Journal of Machine Tools and Manufacture, 48(78), 932-945. 